\title{
Détermination en milieu naturel du dioxide de chlore, des ions chlorite et chlorate basée sur l'utilisation du carmin indigo: étude des interférences \\ Determination of chlorine dioxide, chlorite and chlorate by indigo carmine methods in natural waters: study of interferences
}

\author{
C. Elleouet, F. Quentel et C. L. Madec
}

Volume 12, numéro 3, 1999

URI : https://id.erudit.org/iderudit/705366ar

DOI : https://doi.org/10.7202/705366ar

Aller au sommaire du numéro

Éditeur(s)

Université du Québec - INRS-Eau, Terre et Environnement (INRS-ETE)

ISSN

0992-7158 (imprimé)

1718-8598 (numérique)

Découvrir la revue

Citer cet article

Elleouet, C., Quentel, F. \& Madec, C. L. (1999). Détermination en milieu naturel du dioxide de chlore, des ions chlorite et chlorate basée sur l'utilisation du carmin indigo: étude des interférences. Revue des sciences de l'eau / Journal of Water Science, 12(3), 561-575. https://doi.org/10.7202/705366ar

\section{Résumé de l'article}

Différentes méthodes fondées sur l'exploitation d'un même réactif à savoir le carmin indigo ont été mises en œuvre pour réaliser le suivi du dioxyde de chlore et des sous-produits de dégradation que sont les ions chlorite et chlorate.

L'étude de la stabilité du carmin indigo a permis de montrer que la détermination du dioxyde de chlore doit être effectuée dans les premières heures qui suivent l'ajout de carmin indigo, une légère diminution de l'absorbance étant observée au delà de vingt heures. L'absorbance du carmin indigo en présence d'ions chlorite et chlorate reste en revanche stable plusieurs jours.

La recherche d'éventuelles interférences (substances humiques, ozone, hypochlorite) a également été effectuée. Les ions chlorite et chlorate réagissent avec les substances humiques en milieu acide selon une cinétique réactionnelle beaucoup plus lente que celle des ions chlorite et chlorate sur le carmin indigo. De ce fait, les pourcentages d'erreur sur les concentrations restent faibles. L'hypochlorite ou plus précisément l'acide hypochloreux réagit avec le carmin indigo ce qui conduit à des erreurs dans la détermination du dioxyde de chlore, des ions chlorite et chlorate. Dans le cas du dosage du dioxyde de chlore, les sources d'erreur peuvent être éliminées en ajoutant de l'ammoniaque avant l'introduction du carmin indigo dans l'échantillon.

Après avoir été validés dans des milieux synthétiques, les protocoles ont été appliqués à un milieu naturel : l'eau de distribution de la ville de Brest. Une analyse statistique a été effectuée dans le but de comparer les résultats avec ceux déduits d'autres méthodes basées sur des principes différents. 


\title{
Détermination en milieu naturel du dioxyde de chlore, des ions chlorite et chlorate basée sur l'utilisation du carmin indigo : étude des interférences
}

\author{
Determination of chlorine dioxide, chlorite \\ and chlorate by indigo carmine methods in natural \\ waters: Study of interferences
}

\section{ELLEOUET*', F. QUENTEL et C.L. MADEC}

Reçu le 6 mai 1998, accepté le 28 avril 1999**.

\section{SUMMARY}

Over the last decade, chlorine dioxide has been increasingly used for disinfecting drinking water in many countries. A guarantee for the protection of the consumer is the presence of a sufficient residual concentration of the bactericidal reagent in drinking water. Thus it is important to determine exactly and accurately the levels of chlorine dioxide at the tap. During water treatment and subsequent distribution, chlorine dioxide can undergo a variety of reduction and disproportionation reactions producing primarily chloride but also chlorite and chlorate, which have been shown to cause haemolytic anemia. Reliable analytical methods are needed to identify and determine levels of chlorine dioxide, chlorite and chlorate in drinking water. A procedure based on the use of indigo carmine for the determination of each species in natural waters is suggested in this paper.

In phosphate buffer (pH 6.8), two moles of chlorine dioxide oxidize one mole of indigo carmine. The concentration of the bactericidal reagent can be determined by measuring the difference in absorbance of the dye at $610 \mathrm{~nm}$ before and after reaction with chlorine dioxide. This method is selective as chlorite and chlorate do not react with indigo carmine in phosphate buffer at pH 6.8. Although the spectrophotometric method can be used successfully used at levels of chlorine dioxide down to $30 \mu \mathrm{g} /$, the determination of lower levels in tap water requires a more sensitive method such as an electrochemical stripping procedure. This analysis is based on the measurement of the decrease in the indigo carmine signal after addition of chlorine dioxide. The detection limit is around $1 \mu \mathrm{g} /$.

Laboratoire de Chimie Analytique, UMR 6521, Université de Bretagne Occidentale, 6 av. Victor Le Gorgeu, BP 809, 29285 Brest cedex, France.

* Correspondance. E-mail : Catherine.Elleouet@univ-brest.fr

** Les commentaires seront reçus jusqu'au 10 mai 2000. 
At $\mathrm{pH}=2$, one mole of indigo carmine reduces one mole of chlorite. Thus the chlorite concentration can be determined by measuring the indigo carmine absorbance at $\mathrm{pH}=2$. At $\mathrm{pH}=0$, indigo carmine reacts with both chlorite and chlorate. A measurement at $\mathrm{pH}=\mathbf{0}$ allows chlorate concentrations to be determined since the decrease in absorbance due to the presence of chlorite can be calculated.

The stability of indigo carmine absorbance has been studied. An indigo carmine solution prepared in phosphate buffer is stable over several days if kept in light-proof bottles. It is not surprising that the presence of chlorite and chlorate does not lead to a change in absorbance as they do not react with the dye at $\mathrm{pH}=6.8$. A slight decrease in absorbance of an indigo carmine solution containing chlorine dioxide is observed after about twenty hours. This means that the chlorine dioxide concentration has to be determined in the first hours, which follow the addition of the dye to the sample in order to avoid errors.

Interferences can arise from other residual oxidants, which may also be used in water treatment, or from substances present in the sample, which may react with indigo carmine, chlorite and chlorate. Accordingly, we have considered the influence of humic substances, ozone and hypochlorite. The absorbance of indigo carmine at $\mathrm{pH}=2$ and at $\mathrm{pH}=0$ does not change in presence of natural organic matter $(1 \mathrm{mg} / 1)$. Chlorite and chlorate react with humic substances but the kinetics are much slower than those of the reactions with indigo carmine. Errors arising from humic substances in chlorite and chlorate measurements are thus very weak. Ozone may interfere in analyses as it reacts with indigo carmine. However its existence in the distribution network is unlikely as it also reacts with chlorine dioxide, which is in excess, and chlorite to give chlorate. Hypochlorite causes errors in chlorine dioxide, chlorite and chlorate determinations as a result of a reaction with indigo carmine. In the case of chlorine dioxide determinations, errors can be eliminated by adding ammonia to the sample before indigo carmine.

Once the validity of the procedures had been proven in synthetic media, the methods were applied to a natural water, that of the water distribution network of the city of Brest, France. The results have been compared with those of other analytical techniques.

Key-words: distribution network, chlorine dioxide, chlorite, chlorate, indigo carmine, humic substances, ozone, hypochlorite.

Différentes méthodes fondées sur l'exploitation d'un même réactif à savoir le carmin indigo ont été mises en ouvre pour réaliser le suivi du dioxyde de chlore et des sous-produits de dégradation que sont les ions chlorite et chlorate.

L'étude de la stabilité du carmin indigo a permis de montrer que la détermination du dioxyde de chlore doit être effectuée dans les premières heures qui suivent l'ajout de carmin indigo, une légère diminution de l'absorbance étant observée au-delà de vingt heures. L'absorbance du carmin indigo en présence d'ions chlorite et chlorate reste en revanche stable plusieurs jours.

La recherche d'éventuelles interférences (substances humiques, ozone, hypochlorite) a également été effectuée. Les ions chlorite et chlorate réagissent avec les substances humiques en milieu acide selon une cinétique réactionnelle beaucoup plus lente que celle des ions chlorite et chlorate sur le carmin indigo. De ce fait, les pourcentages d'erreur sur les concentrations restent faibles. L'hypochlorite ou plus précisément l'acide hypochloreux réagit avec le carmin indigo ce qui conduit à des erreurs dans la détermination du 
dioxyde de chlore, des ions chlorite et chlorate. Dans le cas du dosage du dioxyde de chlore, les sources d'erreur peuvent être éliminées en ajoutant de l'ammoniaque avant l'introduction du carmin indigo dans l'échantillon.

Après avoir été validés dans des milieux synthétiques, les protocoles ont été appliqués à un milieu naturel : l'eau de distribution de la ville de Brest. Une analyse statistique a été effectuée dans le but de comparer les résultats avec ceux déduits d'autres méthodes basées sur des principes différents.

Mots clés : réseau de distribution, dioxyde de chlore, chlorite, chlorate, carmin indigo, substances humiques, ozone, hypochlorite.

\section{INTRODUCTION}

Depuis une quinzaine d'années, le dioxyde de chlore est de plus en plus souvent utilisé comme agent bactéricide dans les réseaux de distribution (MASSCHELEIN, 1984). Outre sa propriété d'éviter la formation de trihalométhanes (RAV-ACHA et al., 1984 ; STEVENS, 1982), il possède un pouvoir rémanent élevé. Cependant, suite à diverses réactions chimiques, le dioxyde de chlore peut donner lieu à la formation d'ions chlorite et chlorate. Au fur et à mesure que la concentration de ces espèces augmente dans les canalisations, l'agent bactéricide disparaît. Un résiduel suffisant en dioxyde de chlore dans le réseau étant une garantie pour la protection du consommateur, il est nécessaire de disposer d'une technique fiable et sensible permettant d'accéder aux faibles teneurs comme celles rencontrées dans l'eau prélevée au robinet.

S'il est important de pouvoir déterminer les faibles teneurs en dioxyde de chlore, il est également de l'intérêt de tous de disposer de techniques analytiques capables de discerner tant qualitativement que quantitativement chacune des espèces suivantes : $\mathrm{ClO}_{2}, \mathrm{ClO}_{2}^{-}$et $\mathrm{ClO}_{3}^{-}$. Des études conduites en laboratoire sur le rat tendent en effet à prouver que les deux sous-produits de dégradation peuvent engendrer une diminution du taux d'hémoglobine (CONDIE, 1986 ; DANIEL et al., 1990). En l'absence de norme française, les traiteurs d'eaux adoptent comme référence les recommandations américaines à savoir que la somme $\mathrm{ClO}_{2}+\mathrm{ClO}_{2}^{-}+\mathrm{ClO}_{3}^{-}$doit être inférieure à $1 \mathrm{mg} / \mathrm{l}$.

Le but de ce travail est de proposer un protocole susceptible d'être retenu par les organismes de normalisation pour réaliser le suivi de chacune des espèces. À ce titre, nous avons mis en œuvre, sur la base de données de la littérature, différentes méthodes fondées sur l'exploitation d'un même réactif : le carmin indigo. 


\section{MATÉRIEL ET RÉACTIFS}

\section{Matériel}

Les mesures d'absorbance du carmin indigo seul ou en présence d'espèces oxychlorées sont réalisées à l'aide d'un spectrophotomètre Hitachi U-2010 (cuves en quartz de $5 \mathrm{~cm}$ ou de $1 \mathrm{~cm}$ ).

Un ensemble voltampérométrique PRG5 Tacussel couplé à une électrode à goutte de mercure Metrohm EA 290 est utilisé pour les études de voltamétrie impulsionnelle différentielle (surface de la goutte $1,82 \mathrm{~mm}^{2}, 2$ impulsions par seconde, vitesse de balayage $4 \mathrm{mV} / \mathrm{s}$, hauteur d'impulsion $50 \mathrm{mV}$, durée de l'impulsion $48 \mathrm{~ms})$. Les potentiels sont rapportés à l'électrode $\mathrm{Ag} / \mathrm{AgCl}\left(\mathrm{Cl}^{-} 0,7\right.$ mol/l). L'électrode auxiliaire est un fil de platine.

Les paramètres expérimentaux utilisés en électrophorèse capillaire $(E C)$ et en chromatographie ionique $(\mathrm{Cl})$ sont consignés dans le tableau 1.

Tableau 1 Principaux paramètres utilisés en électrophorèse capillaire $(E C)$ et en chromatographie ionique (Cl).

Table 1 Main parameters of capillany electrophoresis (EC) and ionic chromatography (Cl).

\begin{tabular}{|c|c|c|}
\hline & EC & $\mathrm{Cl}$ \\
\hline Appareillage & $\begin{array}{l}\text { CIA WATERS } \\
\text { capillaire en silice fondue } \\
(75 \mu \mathrm{m} \times 60 \mathrm{~cm})\end{array}$ & $\begin{array}{l}\text { DIONEX DX } 100 \\
\text { boucle d'injection } 50 \mu \\
\text { colonne AS9-SC } \\
\text { suppresseur ASRS-1 }\end{array}$ \\
\hline Eluant & $\begin{array}{l}\text { acide trimellique } 510^{-3} \mathrm{~mol} / \\
\text { OFM } 510^{-4} \mathrm{~mol} / \mathrm{A}\end{array}$ & $\begin{array}{c}\mathrm{NaHCO}_{3} 310^{-4} \mathrm{~mol} / \mathrm{l} \\
\mathrm{Na}_{2} \mathrm{CO}_{3} 310^{-4} \mathrm{~mol} / 1 \\
1 \mathrm{ml} / \mathrm{min}\end{array}$ \\
\hline Détecteur & UV inverse & Conductimétrie \\
\hline $\begin{array}{c}\text { Seuil } \\
\text { de détection }\end{array}$ & $\begin{array}{l}\text { chlorites : } 30 \mu g / 1 \\
\text { chlorates : } 30 \mu g / 1\end{array}$ & $\begin{array}{l}\text { chlorites : } 30 \mu \mathrm{g} / 1 \\
\text { chlorates : } 30 \mu \mathrm{g} / 1\end{array}$ \\
\hline
\end{tabular}

L'acidité des échantillons est contrôlée à l'aide d'un pHmètre Orion 811 équipé d'une sonde de température.

\section{Réactifs}

Toutes les solutions sont préparées à partir d'eau ultra pure (Millipore MilliQ).

Le carmin indigo provient de chez Aldrich (pureté $85 \%$ ) (ref 13,116-4). Une solution mère à $3,4010^{-4} \mathrm{~mol} / /$ est préparée en milieu acide chlorhydrique $0,24 \mathrm{~mol} / \mathrm{l}$. Cette solution peut être conservée plusieurs semaines au réfrigérateur à l'abri de la lumière.

La solution mère de dioxyde de chlore a été préparée selon la réaction suivante (ATOCHEM, 1978):

$$
5\left(\mathrm{Na}^{+}, \mathrm{ClO}_{2}^{-}\right)+2\left(\mathrm{H}^{+}, \mathrm{HSO}_{4}^{-}\right) \rightarrow 4 \mathrm{ClO}_{2}+\left(\mathrm{Na}^{+}, \mathrm{Cl}^{-}\right)+2\left(2 \mathrm{Na}^{+}, \mathrm{SO}_{4}^{2-}\right)+2 \mathrm{H}_{2} \mathrm{O}
$$


Le titre de la solution mère et celui des solutions diluées sont contrôlés par spectrophotométrie UV avant chaque utilisation $\left(\lambda_{\max }=360 \mathrm{~nm}, \varepsilon=1200 \mathrm{~V} \mathrm{~mol} /\right.$ $\mathrm{cm})$.

Les ions chlorite proviennent de chez Aldrich (ref 24,415-5). Ils ont été recristallisés deux fois dans de l'eau chaude afin d'obtenir une pureté supérieure à $96 \%$. Les solutions étalons de chlorite sont fréquemment titrées par iodométrie puis conservées au réfrigérateur.

Les ions chlorate proviennent de chez Merck (ref 6423).

Les solutions mères d'acides humiques sont préparées selon un protocole décrit antérieurement par QUENTEL et al. (1987). Ils proviennent de la rivière Elorn dont l'eau alimente la ville de Brest.

\section{MÉTHODOLOGIE ANALYTIQUE}

\section{Prélèvements}

Un double prélèvement doit être mis en œuvre, l'un pour déterminer la teneur en dioxyde de chlore (a), l'autre pour accéder aux concentrations en chlorite et chlorate (b).

(a) Prélever l'échantillon à doser et l'introduire immédiatement dans une fiole jaugée de $50,0 \mathrm{ml}$ contenant le tampon phosphate $\left(\mathrm{NaH}_{2} \mathrm{PO}_{4} 510^{-3} \mathrm{~mol} / \mathrm{/}\right.$ $\left.\mathrm{Na}_{2} \mathrm{HPO}_{4} 510^{-3} \mathrm{~mol} / / ; \mathrm{pH}=6,8\right)$ et le carmin indigo. La concentration du réactif est choisie en fonction de la quantité de dioxyde de chlore attendue ; elle doit être du même ordre de grandeur. Après avoir bien agité la fiole, le contenu est transvasé dans un flacon inactinique.

(b) Le second échantillon qui est destiné au dosage des ions chlorite et chlorate est immédiatement introduit dans un flacon inactinique puis dégazé par barbotage d'azote de façon à éliminer le dioxyde de chlore résiduel.

\section{Dosage du dioxyde de chlore}

Le dosage du dioxyde de chlore est réalisé par spectrocolorimétrie selon le procédé au carmin indigo mis au point par HOIGNE et BADER (1980). La méthode est sélective, $\mathrm{ClO}_{2}^{-}$et $\mathrm{ClO}_{3}^{-}$étant chimiquement inertes vis-à-vis du réactif en milieu neutre. Les résultats obtenus par ce procédé sont en bon accord avec ceux déduits de la méthode au rouge de chlorophénol (FLETCHER et HEMMINGS, 1985) (écarts inférieurs à $5 \%$ ). La limite de détection est de l'ordre de $30 \mu \mathrm{g} / \mathrm{l}$.

Pour les concentrations inférieures à $30 \mu \mathrm{g} / \mathrm{l}$, la détermination de l'agent bactéricide est effectuée par électrochimie selon le procédé mis au point par QUENTEL et al. (1996). La méthode a été comparée avec une autre méthode ampérométrique utilisant l'oxyde de phénylarsine comme agent titrant (MADEC et al., 1983) et avec un autre procédé électrochimique mettant en jeu le rouge de chlorophénol (LE FUR, 1991). Les trois procédés sont en bon accord. La limite de détection est de l'ordre de $1 \mu \mathrm{g} /$ l. La recherche d'éventuelles interférences a montré d'une part que les produits d'oxydation formés au cours du traitement de l'eau ne modifient pas le signal électrochimique, d'autre part que 
la détermination du dioxyde de chlore n'est pas affectée par la présence des ions chlorite et chlorate aux concentrations rencontrées dans le réseau.

\section{Dosage des ions chlorite et chlorate}

Si les ions $\mathrm{ClO}_{2}^{-}$et $\mathrm{ClO}_{3}^{-}$ne donnent lieu à aucune réaction avec le carmin indigo en milieu neutre, un abaissement sélectif du $\mathrm{pH}$ conduit en revanche à des réactions d'oxydoréduction exploitables à des fins analytiques (CHISWELL et KELLER-LEHMANN, 1993). Ainsi en opérant à $\mathrm{pH}=2$ en présence de fer(II) d'une part et en milieu très acide $(5 \mathrm{~mol} / \mathrm{l})$ d'autre part on accède aux concentrations en chlorite et chlorate. Les conditions expérimentales optimales à respecter et les limites de détection sont rapportées dans le tableau 2.

Tableau 2 Conditions expérimentales optimales pour le dosage des ions chlorite et chlorate par la méthode au carmin indigo.

Table 2 Best experimental conditions for the determination of chlorite and chlorate by the indigo carmine method.

\begin{tabular}{|c|c|c|c|c|c|}
\hline & $\mathrm{pH}$ & $\begin{array}{c}{[\mathrm{Fe}(\mathrm{II})]} \\
(\mathrm{mol} / \mathrm{)})\end{array}$ & $\begin{array}{c}\text { Température } \\
\left({ }^{\circ} \mathrm{C}\right)\end{array}$ & $\begin{array}{c}\text { Temps de chaufiage } \\
(\mathrm{min})\end{array}$ & $\begin{array}{c}\text { Seuil de détection } \\
(\boldsymbol{\mu g} / \mathbf{)})\end{array}$ \\
\hline $\mathrm{ClO}_{2}^{-}$ & 2 & $10^{-6}$ & $45-50$ & 10 & 40 \\
\hline $\mathrm{ClO}_{2}^{-}+\mathrm{ClO}_{3}^{-}$ & $<0$ & & $45-50$ & 5 & 30 \\
\hline
\end{tabular}

La fiabilité de la méthode colorimétrique a pu être mise en évidence par comparaison des résultats avec ceux déduits de deux autres techniques à savoir l'électrophorèse capillaire et la chromatographie ionique dont l'intérêt premier est qu'elles ne mettent pas en jeu de réaction chimique (analyse directe) (BONDOUX et SIEBRAND, 1995 ; DIETRICH et al., 1992).

CHISWELL et KELLER-LEHMANN (1993) n'ayant pas recherché les éventuelles interférences susceptibles d'être rencontrées dans les milieux naturels, ni abordé la stabilité du carmin indigo dans des conditions expérimentales variées, il nous a paru important d'examiner ces différents aspects avant d'exploiter la méthode dans les milieux naturels.

\section{RÉSULTATS ET DISCUSSION}

\section{Stabilité du carmin indigo}

Plusieurs heures peuvent s'écouler entre le moment des prélèvements effectués par les traiteurs d'eaux et celui des analyses. II est donc important d'étudier la stabilité de l'absorbance du carmin indigo en l'absence et en présence de $\mathrm{ClO}_{2}$ d'une part et en présence de $\mathrm{ClO}_{2}{ }^{-}$et $\mathrm{ClO}_{3}{ }^{-}$d'autre part.

La figure 1 reproduit l'évolution de l'absorbance de solutions de carmin indigo préparées en milieu tampon phosphate en fonction du temps. Après 
chaque mesure, les solutions sont replacées à l'abri de la lumière dans un flacon inactinique. La solution de carmin indigo préparée et conservée dans de telles conditions est stable pendant plusieurs jours (courbe a $(O)$ ). La courbe $b$ ( $\square$ ) montre que la présence concomittante de $\mathrm{ClO}_{2}^{-}$et $\mathrm{ClO}_{3}^{-}$n'entraîne pas davantage de variation de l'absorbance. Ce résultat était prévisible dans la mesure où les ions chlorite et chlorate ne réagissent pas avec l'indicateur coloré à $\mathrm{pH}=6,8$. En outre l'absorbance reste constante pendant plusieurs jours. La courbe $c(\Delta)$ relative à la solution de carmin indigo en présence de $\mathrm{ClO}_{2}$ révèle en revanche une très légère diminution de l'absorbance au-delà de vingt heures. La concentration en $\mathrm{ClO}_{2}$ étant calculée par différence des absorbances de la courbe a et de la courbe c, il en résulte que la mesure doit être effectuée dans les premières heures qui suivent l'ajout de carmin indigo dans l'échantillon à doser. Une erreur par excès de $7 \%$ est commise sur la concentration en $\mathrm{ClO}_{2}$ si l'analyse est faite 7 jours après le prélèvement.

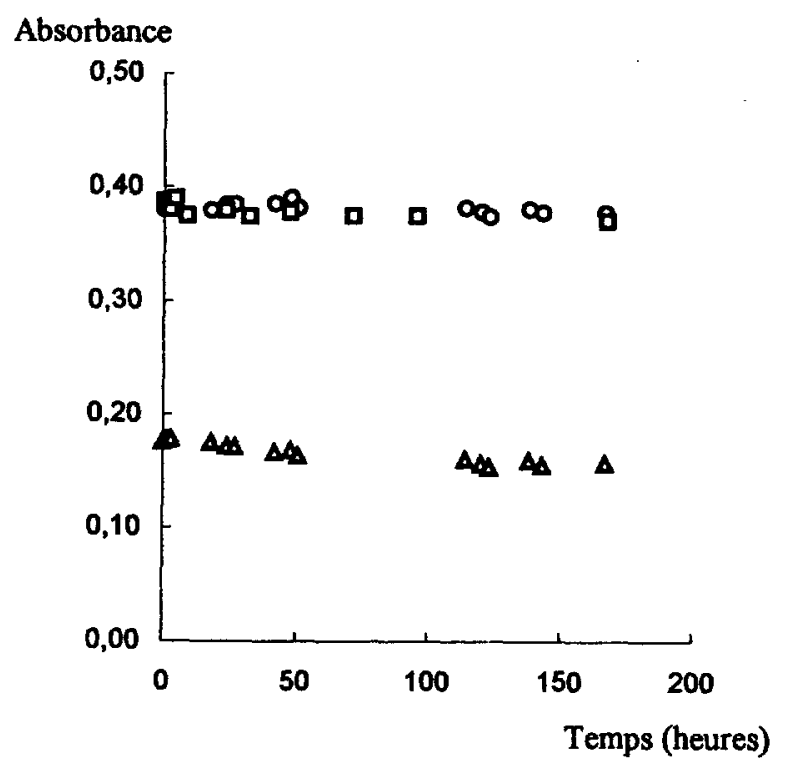

Figure 1 Variation de l'absorbance du carmin indigo en fonction du temps. Variation of the indigo carmine absorbance as a function of time.

\section{Interférences}

Les eaux naturelles peuvent contenir des matières organiques susceptibles d'interagir avec le carmin indigo ainsi qu'avec les ions chlorite et chlorate ; l'eau du réseau de distribution de Brest provenant de l'Elorn, des substances humiques (SH) extraites de cette rivière ont été utilisées pour réaliser les expériences en milieu synthétique. En outre, des traitements simultanés à l'ozone ou à l'hypochlorite peuvent induire des réactions secondaires qu'il convient d'appréhender. 


\section{Substances humiques}

En l'absence de tout traitement chimique préalable, la présence de substances humiques à des teneurs de l'ordre du $\mathrm{mg} / \mathrm{l}$ ne modifie pas l'absorbance de la solution de carmin indigo tant à $\mathrm{pH}=2$ qu'à $\mathrm{pH}=0$.

Les tableaux 3 et 4 regroupent les résultats de dosage de chlorite par le carmin indigo à $\mathrm{pH}=2$ et à $\mathrm{pH}=0$ dans les solutions suivantes :

solution a : eau MilliQ $+\mathrm{ClO}_{2}^{-} 0,300 \mathrm{mg} / \mathrm{l}$

solution $b$ : solution $\mathrm{a}+\mathrm{SH} 1 \mathrm{mg} / \mathrm{l}$

solution $\mathrm{c}$ : solution $\mathrm{b}$ acidifiée au $\mathrm{pH}$ correspondant à celui du dosage et chauffée à $45-50^{\circ} \mathrm{C}$ pendant 10 minutes avant le dosage par ajout de carmin indigo.

Tableau 3 Détermination des ions chlorite $(\mathrm{mg} / \mathrm{l})$ à $\mathrm{pH}=2$ dans différents échantillons.

Table 3 Determination of chlorite $(\mathrm{mg} / \mathrm{l})$ at $\mathrm{pH}=2$ in different samples.

\begin{tabular}{|l|c|c|c|}
\hline & Absorbance à $\mathbf{~} \mathrm{H}=2$ & {$\left[\mathrm{ClO}_{2}^{-}\right]$calculée } & $\%$ d'erreur \\
\hline solution a & 1,07 & 0,318 & \\
\hline solution b & 1,10 & 0,299 & 6 \\
\hline solution c & 1,13 & 0,280 & 12 \\
\hline
\end{tabular}

Tableau 4 Détermination des ions chlorite $(\mathrm{mg} / \mathrm{l})$ à $\mathrm{pH}=0$ dans différents échantillons.

Table 4 Determination of chlorite $(\mathrm{mg} / \mathrm{l})$ at $\mathrm{pH}=\mathrm{O}$ in different samples.

\begin{tabular}{|c|c|c|c|}
\hline & Absorbance à $\mathbf{p H}=\mathbf{0}$ & {$\left[\mathrm{ClO}_{\mathbf{2}}^{-}\right]$calculée } & \% d'erreur \\
\hline solution a & 1,13 & 0,300 & \\
\hline solution b & 1,19 & 0,257 & 14 \\
\hline solution c & 1,45 & 0,071 & 76 \\
\hline
\end{tabular}

L'analyse des données montre que les ions chlorite réagissent avec les substances humiques en milieu acide selon une cinétique réactionnelle beaucoup plus lente que celle des ions chlorite sur le carmin indigo. De ce fait les pourcentages d'erreur, sur la concentration en chlorite retrouvée en présence de substances humiques par rapport à celle calculée dans le cas d'une solution exempte de matière organique, restent faibles $(6 \%$ à $\mathrm{pH}=2$ et $14 \%$ à pH $=0$ dans le cas présent). L'expérience réalisée avec la solution $\mathrm{c}$ ne traduit pas la réalité du protocole expérimental dans la mesure où l'ajout de carmin indigo a été réalisé après avoir préalablement acidifié et chauffé à $45-50^{\circ} \mathrm{C}$ pendant 10 minutes l'échantillon à doser. Elle a été effectuée dans le but d'accélérer la cinétique de réaction entre les espèces oxychlorées et la matière organique. 
Comme pour les ions chlorite, la présence de substances humiques conduit à une légère diminution du taux de chlorate, résultat d'une réaction à cinétique lente entre ces derniers et la matière organique (tableau 5).

Tableau 5 Détermination des ions chlorate $(\mathrm{mg} / \mathrm{l})$ à $\mathrm{pH}=0$ dans différents échantillons.

Table 5 Determination of chlorate $(\mathrm{mg} / \mathrm{l})$ at $\mathrm{pH}=0$ in different samples.

\begin{tabular}{|l|c|c|c|}
\hline & Absorbance à pH = & {$\left[\mathrm{ClO}_{3}^{-}\right]$calculée } & \% d'erreur \\
\hline solution a & 1,04 & 0,291 & \\
\hline solution b & 1,11 & 0,251 & 14 \\
\hline solution c & 1,40 & 0,086 & 70 \\
\hline
\end{tabular}

solution a : eau MilliQ $+0,300 \mathrm{mg} /$

solution b : solution $\mathrm{a}+\mathrm{SH} 1 \mathrm{mg} / \mathrm{I}$

solution $\mathrm{c}$ : solution b acidifiée à $\mathrm{pH}=0$ et chauffée à $45-50^{\circ} \mathrm{C}$ pendant

10 minutes avant le dosage par ajout de carmin indigo.

L'ozonation constitue une étape importante du traitement de l'eau de distribution de la Communauté Urbaine de Brest. II nous a donc semblé important de vérifier que la matière organique soumise à un tel traitement ne conduit pas à la formation d'espèces susceptibles d'interagir avec le carmin indigo, les ions chlorite et chlorate lors de l'acidification du milieu. Les analyses effectuées en présence de substances humiques préalablement ozonées conduisent aux mêmes résultats que ceux obtenus précédemment. Il apparaît donc que les substances humiques " ozonées " ne réagissent pas avec le carmin indigo et conservent vis-à-vis des ions chlorite et chlorate la même interaction que celles n'ayant pas subi le traitement.

\section{Ozone}

L'ozone devrait interférer dans le dosage du dioxyde de chlore puisqu'il réagit avec le carmin indigo (BADER et HOIGNE, 1981). Mais sa réactivité vis-à-vis de $\mathrm{ClO}_{2}$ et $\mathrm{ClO}_{2}^{-}$pour produire $\mathrm{ClO}_{3}^{-}$rend incompatible la coexistence de $\mathrm{O}_{3}$, $\mathrm{ClO}_{2}$ et $\mathrm{ClO}_{2}^{-}$(HOIGNE et al., 1985). La présence d'ozone à la sortie d'une usine utilisant le dioxyde de chlore comme agent bactéricide est donc improbable, celui-ci étant en excès par rapport à $\mathrm{O}_{3}$. En effet, pour éviter la transformation de $\mathrm{ClO}_{2}$ en $\mathrm{ClO}_{2}^{-}$et donc la perte du pouvoir bactéricide, une étape de " déozonation " sur cascade est préalablement mise en œuvre.

\section{Hypochlorite}

Certaines usines ont recours à la fois au dioxyde de chlore et à l'hypochlorite dans l'étape de désinfection. Contrairement à $\mathrm{O}_{3}, \mathrm{ClO}^{-}$fou $\mathrm{HClO}$ selon le $\mathrm{pH}$ du milieu) peut coexister avec $\mathrm{ClO}_{2}$, la cinétique réactionnelle étant lente (KARPEL VEL LEITNER et al., 1994). 


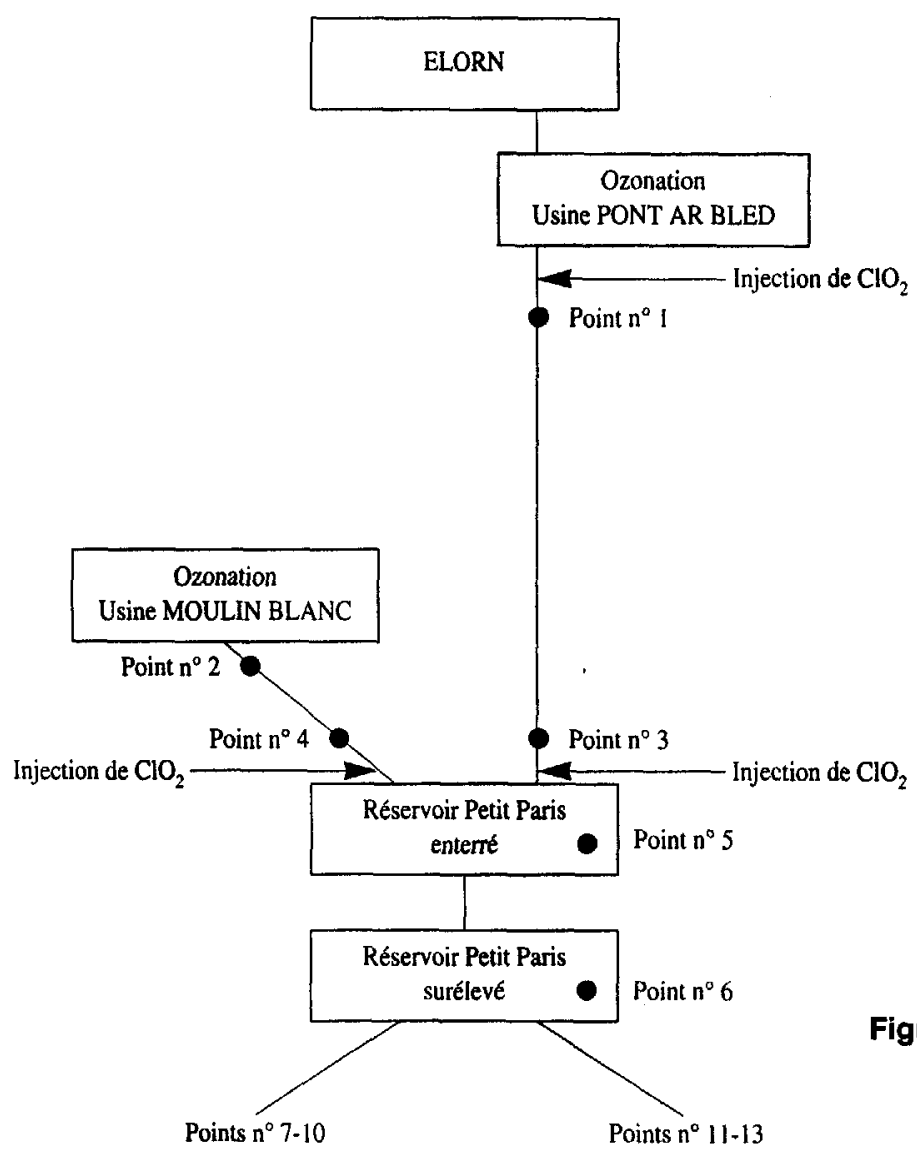

\begin{tabular}{|c|c|}
\hline Dénomination & Caractéristiques \\
\hline Point $n^{\circ} 1$ & $\begin{array}{l}\text { Eau traitce ozonce } \\
\text { sortie de l'usine Pont ar Bled } \\
\text { point très proche de l'injection de } \mathrm{ClO}_{2}\end{array}$ \\
\hline Point $n^{\circ} 2$ & $\begin{array}{l}\text { Eau ozonce } \\
\text { sortic de l'usine du Moulin Blanc } \\
\text { pas d'injection de } \mathrm{ClO}_{2}\end{array}$ \\
\hline Point $n^{\circ} 3$ & $\begin{array}{l}\text { Eau désinfectée au } \mathrm{ClO}_{2} \\
\text { sortie de l'usine de Pont ar Bled } \\
\text { point trts cloigne de la } 1^{\text {ton }} \text { injection de } \\
\mathrm{ClO}_{2}\end{array}$ \\
\hline Point $n^{\circ} 4$ & $\begin{array}{l}\text { Eau non désinfectée au } \mathrm{ClO}_{2} \\
\text { sortie de l'usine du Moulin Blanc }\end{array}$ \\
\hline Point n's & $\begin{array}{l}\text { Eau provenant de l'usine de Pont ar Bled } \\
\text { point proche des injections de } \mathrm{ClO}_{2}\end{array}$ \\
\hline Point $n^{\circ} 6$ & $\begin{array}{l}\text { Eau provenant à la fois de l'usine de Pont ar } \\
\text { Bled et de l'usine du Moulin Blanc } \\
\text { point plus éloigné des injections de } \mathrm{ClO}_{2}\end{array}$ \\
\hline Points $n^{\circ} 7-13$ & Divers points du réseau urbain \\
\hline
\end{tabular}

Figure 2 Scheme of the distribution network of the city of Brest. Characteristics of different waters.

Schéma du réseau de distribution de la ville de Brest. Caractéristiques des eaux prélevées. 
En milieu neutre (tampon phosphate) le carmin indigo est oxydé et / ou chloré par l'hypochlorite ou plus précisément par l'acide hypochloreux. Cependant il est possible d'inhiber cette réaction en ajoutant de l'ammoniaque, avant le carmin indigo, dans l'échantillon contenant $\mathrm{ClO}^{-}$(la concentration de l'ammoniaque est environ 5 fois supérieure à celle de l'hypochlorite). II y a ainsi formation de monochloramine qui n'interfère pas dans le dosage du dioxyde de chlore.

$$
\mathrm{NH}_{4}^{+}+\mathrm{HClO} \rightarrow \mathrm{NH}_{2} \mathrm{Cl}+\mathrm{H}_{3} \mathrm{O}^{+}
$$

Il est important de noter que la différence entre les absorbances mesurées à $\mathrm{pH}$ 6,8 en l'absence et en présence d'ammoniaque permet d'accéder à la concentration en acide hypochloreux.

L'acide hypochloreux réagissant également avec le carmin indigo en milieu acide interfère dans la détermination des ions chlorite et chlorate. L'ajout d'ammoniaque ne permet malheureusement pas de résoudre ce problème comme dans le cas du dosage du dioxyde de chlore, la monochloramine formée réagissant avec le carmin indigo en milieu acide. Pour accéder sans erreur aux concentrations en ions chlorite et chlorate, il est alors nécessaire de retrancher aux absorbances mesurées à pH 2 et à pH 0 la part imputable à l'acide hypochloreux dont le taux est déterminé à $\mathrm{pH}$ 6,8 selon le protocole décrit précédemment.

\section{Étude en milieu naturel}

Après avoir vérifié la validité des méthodes dans des milieux synthétiques, nous les avons appliquées à un milieu naturel à savoir l'eau de distribution de la Communauté Urbaine de Brest. Comme le montre le schéma de la figure 2, des prélèvements ont été effectués en divers points du réseau.

Les résultats obtenus sont rassemblés dans le tableau 6. L'eau du Moulin Blanc n'ayant pas subi de traitement au dioxyde de chlore au niveau de l'usine, il n'est pas surprenant de constater l'absence d'ions chlorite et chlorate aux points 2 et 4 . La teneur élevée en ions chlorate dès le point 1 se justifie par une préoxydation avant l'étape d'ozonation au niveau de l'usine. L'augmentation de la concentration en ions chlorite observée entre le point 1 et le point 3 résulte de la dégradation de l'agent bactéricide dans le réseau par suite de réactions chimiques. Bien que l'eau du Moulin blanc et l'eau de Pont ar Bled arrivent toutes deux dans le réservoir Petit Paris enterré, des études de flux ont montré qu'elles ne s'y mélangent pas. Le mélange s'effectue uniquement dans le réservoir surélevé. La comparaison des teneurs mesurées aux points 3 et 5 confirme le fait que l'eau prélevée au point 5 est principalement celle provenant de Pont ar Bled. La diminution des concentrations entre le point 5 et le point 6 est le résultat de la dilution due au mélange des eaux.

Dans le but de comparer les résultats fournis par les trois techniques, une analyse statistique a été effectuée selon le modèle préconisé par BESNARD et MORIN (1997) en prenant la chromatographie ionique comme méthode de référence. Cette analyse révèle une liaison linéaire entre la chromatographie ionique et l'électrophorèse capillaire d'une part, entre la chromatographie ionique et la colorimétrie d'autre part. Les droites de régression ajustées selon les méthodes des moindres carrés sont représentées sur les graphes de la figure 3. L'exploitation de tests statistiques montre que la droite de régression représentée sur le 
Tableau 6 Détermination du dioxyde de chlore, des ions chlorite et chlorate en différents points du réseau de distribution.

Table 6 Determination of chlorine dioxide, chlorite and chlorate at different sampling stations of the distribution network.

\begin{tabular}{|c|c|c|c|c|c|c|c|c|}
\hline & \multicolumn{3}{|c|}{$\mathrm{ClO} 0_{2}^{-}(\mathrm{mg} / \mathrm{l})$} & \multicolumn{3}{c|}{$\mathrm{ClO}_{3}^{-}(\mathrm{mg} / \mathrm{l})$} & \multicolumn{2}{c|}{$\mathrm{ClO}_{2}(\mu \mathrm{g} / \mathrm{l})$} \\
\cline { 2 - 8 } Point & $\mathrm{CL}$ & $\mathrm{EC}$ & $\mathrm{Cl}$ & $\mathrm{CL}$ & $\mathrm{EC}$ & $\mathrm{Cl}$ & $\mathrm{CL}$ & Electro \\
\hline 1 & 0,08 & 0,07 & 0,04 & 0,45 & 0,42 & 0,44 & 280 & \\
2 & 0,03 & 0,00 & 0,00 & 0,00 & 0,00 & 0,00 & & \\
3 & 0,28 & 0,25 & 0,28 & 0,47 & 0,43 & 0,45 & & 40 \\
4 & 0,00 & 0,00 & 0,00 & 0,00 & 0,00 & 0,00 & & \\
5 & 0,25 & 0,25 & 0,30 & 0,43 & 0,38 & 0,37 & 360 & \\
6 & 0,18 & 0,22 & 0,25 & 0,23 & 0,16 & 0,19 & 140 & 13 \\
7 & 0,20 & 0,23 & 0,25 & 0,16 & 0,16 & 0,13 & & 13 \\
8 & 0,16 & 0,17 & 0,19 & 0,11 & 0,10 & 0,12 & & 8 \\
9 & 0,18 & 0,22 & 0,24 & 0,11 & 0,10 & 0,12 & & 10 \\
10 & 0,21 & 0,26 & 0,27 & 0,14 & 0,10 & 0,14 & & 36 \\
11 & 0,18 & 0,23 & 0,26 & 0,11 & 0,11 & 0,14 & & 22 \\
12 & 0,28 & 0,33 & 0,35 & 0,21 & 0,21 & 0,21 & & \\
13 & 0,23 & 0,22 & 0,25 & 0,17 & 0,09 & 0,14 & & 24 \\
$1^{*}$ & 0,02 & 0,04 & 0,06 & 0,46 & 0,36 & 0,43 & 220 & \\
$2^{*}$ & 0,00 & 0,00 & 0,00 & 0,00 & 0,00 & 0,00 & & 30 \\
$3^{*}$ & 0,21 & 0,22 & 0,26 & 0,42 & 0,38 & 0,39 & & 30 \\
$4^{*}$ & 0,00 & 0,00 & 0,00 & 0,00 & 0,00 & 0,00 & & \\
$5^{*}$ & 0,33 & 0,32 & 0,33 & 0,56 & 0,40 & 0,49 & 380 & \\
$6^{*}$ & 0,28 & 0,32 & 0,31 & 0,35 & 0,24 & 0,29 & 190 & \\
$7^{\star}$ & 0,36 & 0,42 & 0,40 & 0,28 & 0,20 & 0,24 & & 22 \\
$8^{*}$ & 0,22 & 0,26 & 0,24 & 0,16 & 0,10 & 0,13 & & 34 \\
$9^{*}$ & 0,27 & 0,34 & 0,35 & 0,29 & 0,16 & 0,23 & & 8 \\
$10^{*}$ & 0,18 & 0,23 & 0,23 & 0,19 & 0,10 & 0,14 & & 14 \\
$11^{*}$ & 0,32 & 0,40 & 0,36 & 0,25 & 0,20 & 0,20 & & 35 \\
$12^{*}$ & 0,34 & 0,44 & 0,42 & 0,27 & 0,19 & 0,24 & & 28 \\
$13^{*}$ & 0,16 & 0,15 & 0,17 & 0,13 & 0,08 & 0,13 & & 26 \\
\hline
\end{tabular}

*série de mesures d'une $2^{\mathrm{e}}$ campagne

$\mathrm{CL}$ : méthode colorimétrique au carmin indigo ; $\mathrm{EC}$ : électrophorèse capillaire ; $\mathrm{Cl}$ : chromatographie ionique ; Electro : méthode électrochimique au carmin indigo

graphe a ne diffère pas de la droite identité $(p=0,05)$. L'électrophorèse capillaire et la chromatographie ionique, appliquées au dosage des ions chlorite, sont donc deux techniques concordantes. En ce qui concerne la droite de régression du graphe $b$, les tests indiquent que l'ordonnée à l'origine n'est pas significativement différente de 0 alors que la pente est statistiquement différente de 1 ( $p<0,0004)$; la colorimétrie induirait donc une erreur relative par défaut d'environ $17 \%$. L'analyse statistique menée, selon le même modèle, pour comparer les concentrations en ions chlorate met en évidence que la colorimétrie mais aussi l'électrophorèse capillaire diffèrent toutes deux de la chromatographie ionique. Elles conduisent chacune à une erreur relative de $8 \%$. II est difficile, en l'état actuel des travaux, d'attribuer les écarts observés à des erreurs systématiques liées à la méthode elle-même ou à la présence dans le réseau d'espèces interférentes. II conviendrait d'effectuer de nouvelles campagnes, dans le réseau de la ville de Brest mais aussi dans d'autres réseaux, pour tenter d'obtenir des éléments de réponse. 


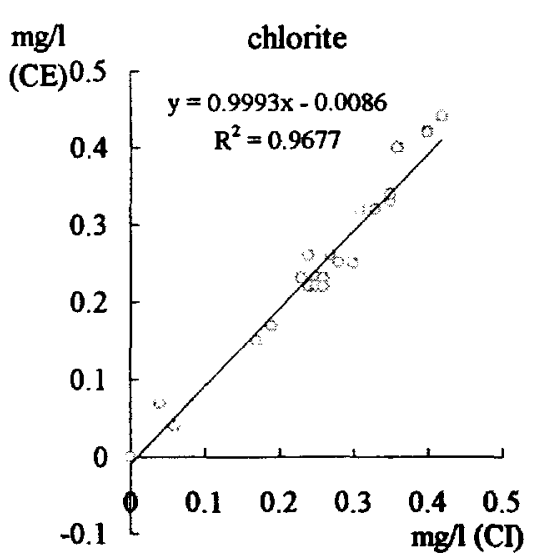

a

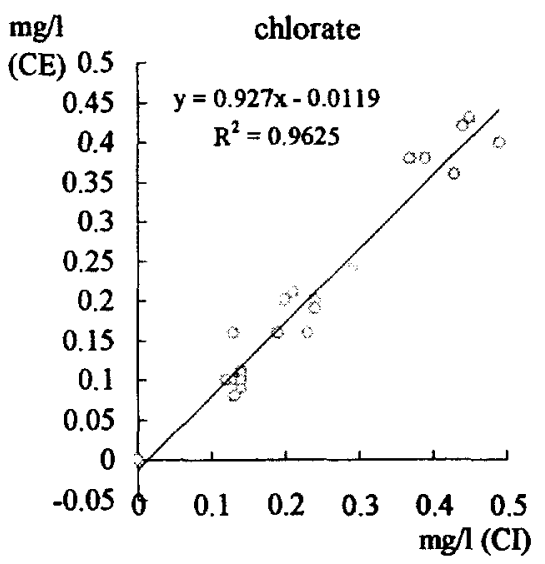

c mg/l chlorite

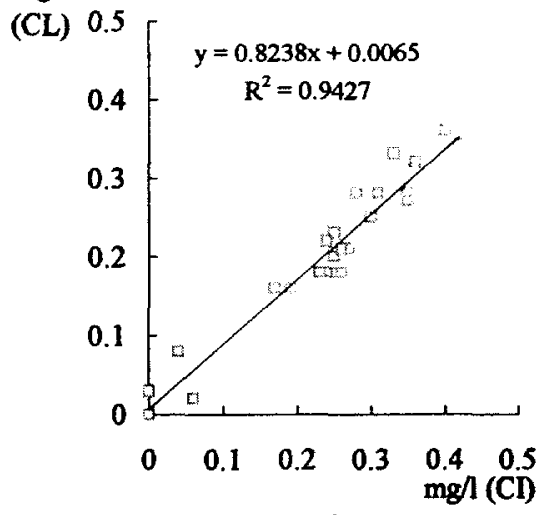

b

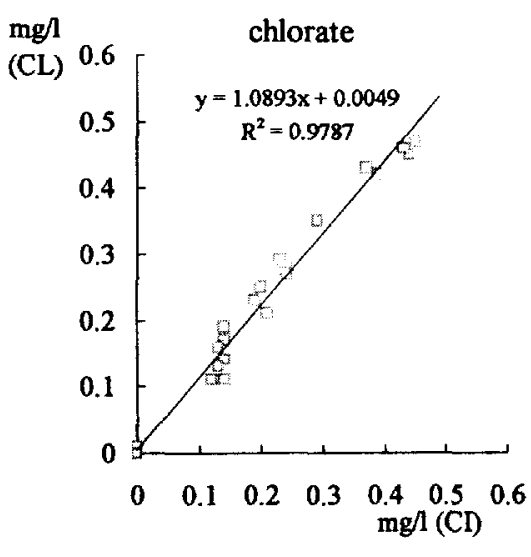

d

Figure 3 Concentrations obtenues par l'électrophorèse capillaire (EC) (a et c) et par la méthode colorimétrique $(\mathrm{CL}$ ) (b et d) comparées à celles de la chromatographie ionique (Cl) prise comme référence.

Concentrations measured by capillary electrophoresis (EC) (a et c) and by the colorimetric method (CL) (b et d) compared with those obtained by ion chromatography (CI) as the reference method.

Quoi qu'il en soit les conclusions statistiques de ce premier travail ne remettent pas en cause l'intérêt de la méthode au carmin indigo dans la mesure où la principale préoccupation des traiteurs d'eau est de pouvoir rapidement et à moindre coût vérifier que la somme $\mathrm{ClO}_{2}+\mathrm{ClO}_{2}^{-}+\mathrm{ClO}_{3}^{-}$ne dépasse pas $1 \mathrm{mg} / \mathrm{l}$. Conscients des erreurs commises ils pourront juger de la nécessité ou pas de faire appel à la chromatographie ionique, technique plus onéreuse. 


\section{CONCLUSION}

L'intérêt du carmin indigo est à appréhender tant du point de vue scientifique que financier. Grâce à ses propriétés colorimétriques, les traiteurs d'eau disposent d'un outil analytique simple et peu onéreux, comparé à l'électrophorèse capillaire et à la chromatographie ionique, leur permettant d'accéder avec précision à la concentration de l'agent bactéricide $\mathrm{ClO}_{2}$ et à l'ordre de grandeur des concentrations résiduelles des sous-produits de dégradation $\mathrm{ClO}_{2}^{-}$et $\mathrm{ClO}_{3}^{-}$ rencontrées dans les réseaux d'eau potable traités à l'agent oxychloré. En outre, grâce à son caractère électroactif sur mercure, il permet d'accéder aux très faibles teneurs en dioxyde de chlore susceptibles d'être rencontrées en fin de réseau c'est-à-dire à la sortie du robinet du consommateur.

\section{REMERCIEMENTS}

Les auteurs remercient la société « Compagnie de l'Eau et de l'Ozone " et plus particulièrement Monsieur Friand pour leur colloboration à la collecte des échantillons.

\section{RÉFÉRENCES BIBLIOGRAPHIQUES}

ATOCHEM CAL, 1978. Centre d'Application de Levallois, ref. LCK 1319.

BADER H., HOIGNE J., 1981. Determination of ozone in water by the indigo method. Water Research, 15, 449-456.

BESNARD J.C., MORIN J.F., 1997. Immuno Stat, NUCLEON, Paris.

BONDOUX G., SIEBRAND A., 1995. Dosage d'anions dans les eaux par électrophorèse capillaire; cas des oxydes de chlore et des bromates. Chimie Nouvelle, 13(52), 1517-1522.

CHISWELL B., KELLER-LEHMANN B., 1993. Spectrophotometric method for the determination of chlorite and chlorate. Analyst, 118, 1457-1459.

CONDIE L. W., 1986. Toxicological problems associated with chlorine dioxide. J. Am. Water Works Assoc., 73-78.

DANIEL F.B., CONDIE L.W., ROBINSON M., STOBER J.A., YORK R.G., OLSON G.R.,
SHIN RU WANG, 1990. Comparative subchronic toxicity studies of three disinfectants. J. Am. Water Works Assoc., 82(70), 61-69.

DIETRICH A.M., LEDDER T.D., GALLAGHER D.L., GRABEEL-M.N., HOEHN R.C., 1992. Determination of chlorite and chlorate in chlorinated and chloraminated drinking water by flow injection analysis and ion chromatography. Anal. Chem., $64,496-502$.

FLETCHER I.J., HEMMINGS P., 1985. Determination of chlorine dioxide in potable waters using chlorophenol red. Analyst, 110, 695-699.

HOIGNE J., BADER H., 1980. Bestimmung von ozon und chlordioxid in wasser mit der indigo-methode. Vom Wasser, 55. 261-279.

HOIGNE J., BADER H., HAAG W.R., STAEHELIN J., 1985. Rate constants of reactions of ozone with organic and inorganic 
compounds in water-lil. Water Research, 19(8), 993-1004.

KARPEL VEL LEITNER N., DE LAAT J., DORE M., SUTY H., POUILLOT M., 1994. Évolution des concentrations en chlorite et en chlorate au sein des filières de potabilisation comprenant une préoxydation au dioxyde de chlore. T. S. M. L'EAU, 1, 27-33.

LE FUR E., 1991. Dosage de traces de dioxyde de chlore en présence de chlorite : exploitation des techniques électrochimiques. Rapport de DEA. Univ. Brest.

MADEC C., TREBERN B., CABON J.Y., COURTOT-COUPEZ J., 1983. Principales méthodes de dosage des oxydants résiduels produits par la chloration des milieux naturels. Analusis, 11(8), 363-379.

MASSCHELEIN W.J., 1984. Experience with chlorine dioxide in Brussels: Generation of chlorine dioxide. J. Am. Water Works Assoc., 70-76.

QUENTEL F., MADEC C., COURTOT-COUPEZ J., 1987. Determination of humic substances in seawater by electrochemistry (mechanisms). Anal. Letters, 20(1), 47-62.

QUENTEL F., ELLEOUET C., MADEC C.L., 1996. Determination of trace levels of chlorine dioxide in drinking water by electrochemistry. Analusis, 24, 199-203.

RAV-ACHA Ch., SERRI A., CHOSHEN (GOLDSTEIN) E., LIMONI B., 1984. Disinfection of drinking water rich in bromide with chlorine and chlorine dioxide, while minimizing the formation of undesirable byproducts. Wat. Sci. Tech., 17, 611-621.

STEVENS A.A., 1982. Reaction products of chlorine dioxide. Environmental Health Perspectives, 46, 101-110. 